\title{
Accuracy of Computer Aided Diagnosis in Predicting Malignant Thyroid Nodule
}

\section{Pandit $\mathrm{SP}^{1 *}$, Thapa $\mathrm{P}^{2}$ and Pandit $\mathrm{S}^{3}$}

${ }^{1}$ Professor of Radio-diagnosis \& imaging, NAMS, Nepal

${ }^{2}$ Medical officer Man Mohan cardiac center, IOM, Nepal

${ }^{3}$ Medical officer, NAMS, Nepal

*Corresponding author: Dr Swoyam Pandit, National Academy of Medical Science (NAMS),

\section{Research Article}

Volume 5 Issue 1

Received Date: March 06, 2021

Published Date: March 26, 2021

DOI: $10.23880 /$ crij-16000182 Bir Hospital, Kathmandu Nepal, Email: swoyampandit@hotmail.com

\section{Abstract}

Introduction: The thyroid nodule is an abnormal growth of thyroid cells which forms a lump within the thyroid gland. The vast majority of thyroid nodules are benign \& a small proportion may have malignancy. It may have solitary or multiple, unilateral or bilateral, focal or diffuse enlargement. The physical examination \& blood test only are not helpful to detect benign from malignant nodules \& needs thyroid ultrasonography and fine needle biopsy for early detection \& treatment. In a suspicious cases, FNA is needed to confirmed benign, from malignant nodules. CAD is a kind of software used in the Sonographic image of thyroid nodule to differentiate benign from malignant condition. CAD software, is non- invasive, no ionizing radiation procedure. It recommend follow up study for benign \& FNA for suspicious of malignancy.

Objective: To assess the clinical use of Computer Aided Diagnosis (CAD) software to correlate the biopsy report with thyroid nodules.

Method: The prospective analytical study was conducted at end of 2018 in department of radio-diagnosis \& imaging in tertiary health care system, Nepal in 103 patients of 128 nodules who were presented with thyroid nodule, Routine with Color Doppler ultrasound was done. Computer aided diagnosis (CAD) software applied \& guided FNA from different nodules were obtained \& correlated with Cyto-pathology study.

Result: A total of 103 patients, male $26(24 \%)$ \& female $78(76 \%)$ in the age group of 20-82 years having a solitary or multiple nodules in unilateral or bilateral thyroid lobe (128 nodules) were evaluated. A total of $87.34 \%$ were benign $\& 12.66 \%$ were malignant nodule Most of the benign nodule was colloid goiter \& commonest neoplasm was papillary carcinoma, which is similar to CAD recommendation. CAD can predict the nature of thyroid nodule as benign or malignant with the sensitivity of 94\% \& specificity $88 \%$ \& PPV $47 \%$ as a compare with FNAC (Gold standard).

Conclusion: CAD effectively \& objectively helps to determine which nodule needs to take FNA from different nodules in the same patient whenever needed.

Keywords: Computer aided diagnosis; Fine-needle aspiration; Thyroid nodule; USG

Abbreviations: CAD: Computer aided diagnosis; ATA:

American Thyroid association. 


\section{Introduction}

Thyroid anatomy: The thyroid gland is one of the endocrine gland consists of two lateral lobes united by isthmus located anterior to the trachea [1]. It Weighs about 15-25 $\mathrm{g}$ in an adults. The thyroid lobes measures about $4.0 \mathrm{~cm} \mathrm{x} 1.5 \mathrm{~cm} \mathrm{x}$ $2.0 \mathrm{~cm}$. The isthmus measure about 4.0-6.0 mm. It has major role in the body metabolism. Computer aided diagnosis (CAD) devices, are intended to provide presence or absence of diseases \& severity of the diseases which has ability to differentiate benign from malignant diseases automatically [2]. Thyroid nodules occur in up to $50 \%$ of an adult, whereas palpable thyroid nodules occur in only 3\%-7\%. Malignancy occurs only in $5 \%-7 \%$ of all thyroid nodules [3]. Thyroid nodules and thyroid malignancy has a female predilection of $4: 1$ and 2.3:1 respectively. In general, the probability of malignancy in a nodule is higher for men and for patients under 15 years or over 45 years of age [4].

Thyroid Ultrasonography: Thyroid ultrasound is a primary tool for thyroid nodule evaluation. This is very accurate, easily available \& determines the solid or cystic nodule and size of the nodule. In USG the thyroid tissue appears homogeneously echogenic with a uniform echo texture. A nodule is generally characterized as hypo- echoic, isoechoic, or hyper-echoic but hypoechogenicity is associated with thyroid malignancy [5]. Most of the thyroid nodules have various internal echogenicity, making it difficult to make the definite diagnosis [6].

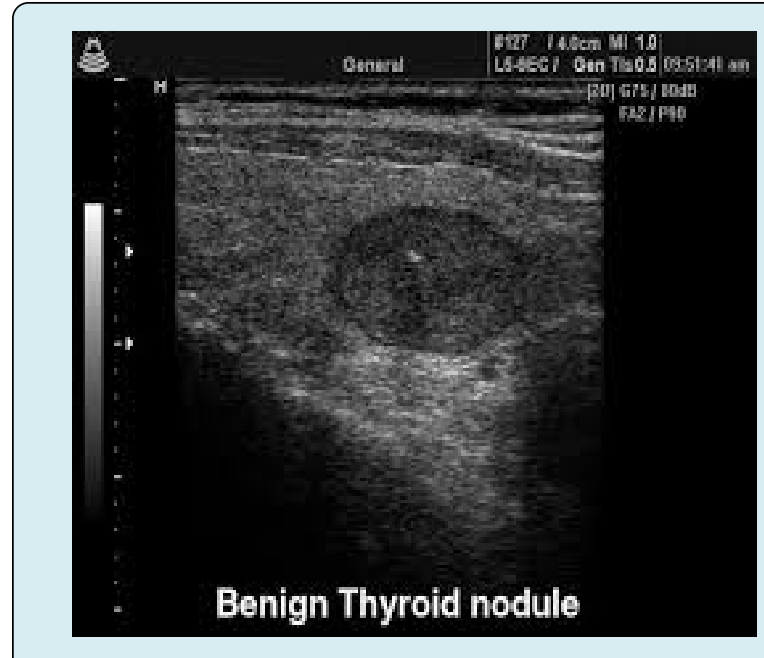

Figure1: Benign nodule in USG.

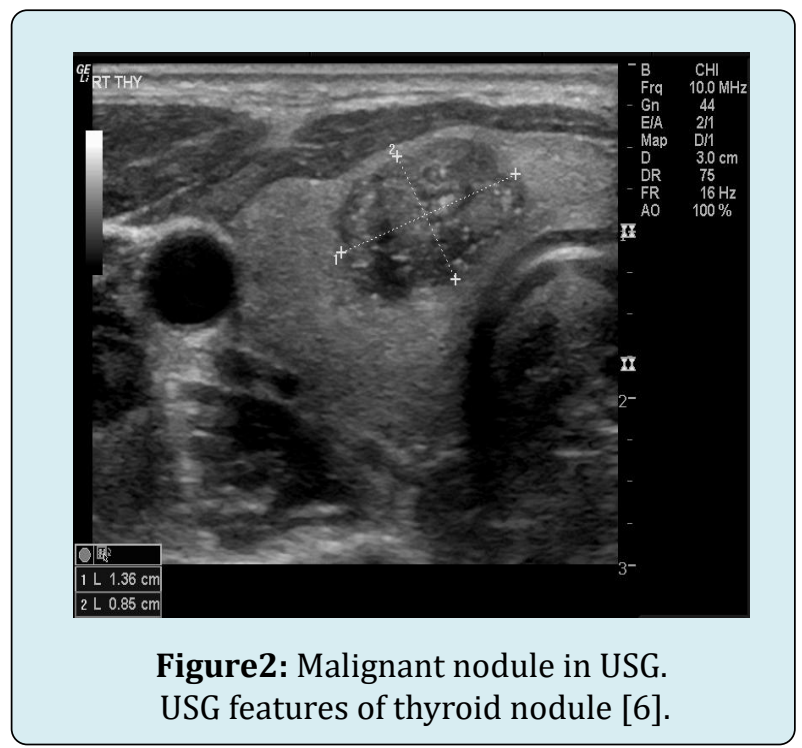




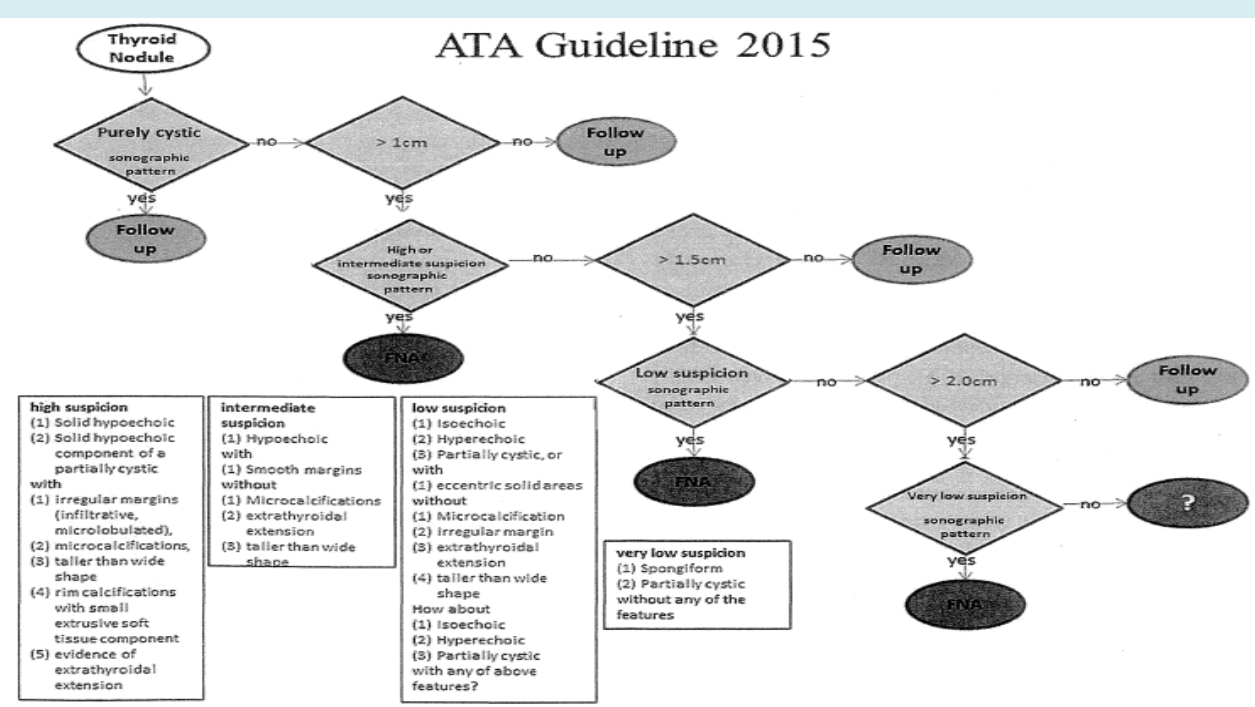

Fig. 1 A diagnostic algorithm from the 2015 ATA guidelines for evaluation and management of patients with thyroid nodules based on ultrasound patterns.

Figure 3: A diagnostic algorithm from the 2015 ATA guidelines for evaluation and management of patients with thyroid nodules based on ultrasound patterns.

\section{What Is Computer Aided Diagnosis (CAD)}

Computer-aided diagnosis (CAD) is a technology being newly developed for characterization of thyroid lesions on USG. It is software used in the Sonographic image of thyroid nodule to differentiate benign from malignant condition [4]. It is a convenient method to determine the necessity of FNA, but is not replace of FNAC. It is also not an examiner dependent measurement. It can differentiate benign from malignant diseases, automatically [7].

\section{Objectives}

To assess the clinical use of Computer Aided Diagnosis (CAD) software to correlate the biopsy report with USG thyroid nodules.

\section{Materials and Methods}

Method-The prospective analytical study was conducted in the 2017-2018 in department of radio-diagnosis \& imaging in tertiary health care system, Nepal in 103 patients of 128 nodules with written consent, who were presented with thyroid nodule. Routine \& Color Doppler ultrasound with Hitachi-Aloka USG machine using linear probe $5-7 \mathrm{MHz}$ was performed. Computer aided diagnosis (CAD) software applied \& guided FNA from different nodules were obtained \& correlated with Cytopathology study. Statistical analysis was done using SPSS version16.0.
Inclusion criteria: All refer patients from OPD who had thyroid nodule above the age of 15 years.

Exclusion criteria: All diagnosed \& under treatment case of thyroid nodule.

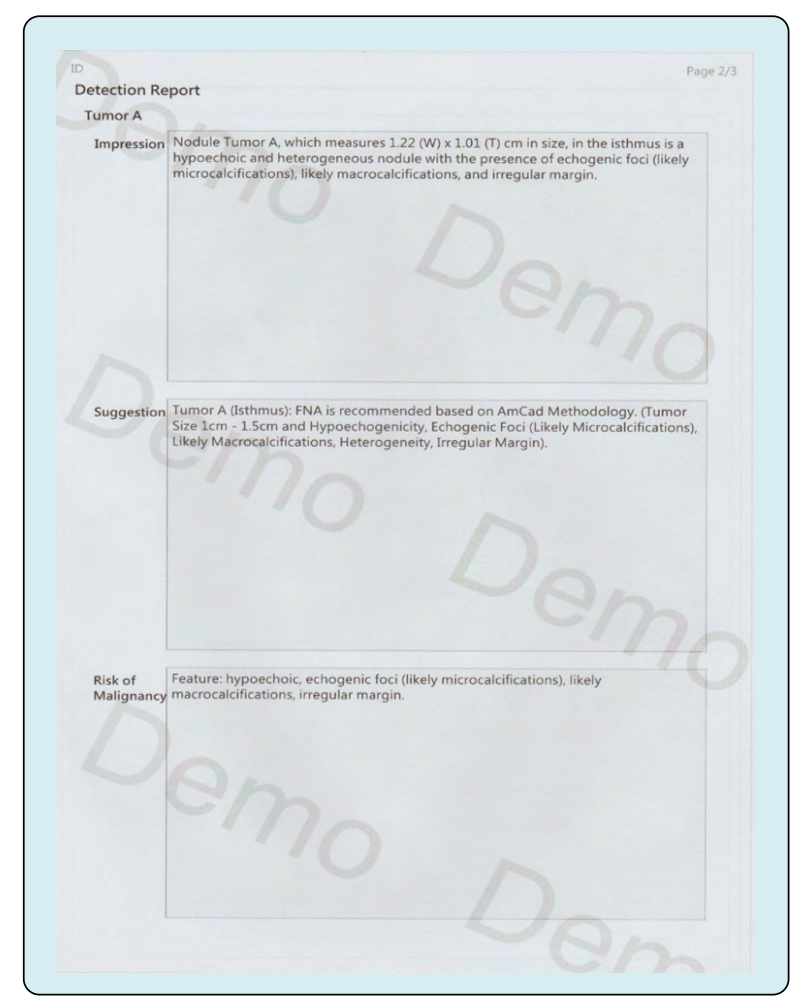




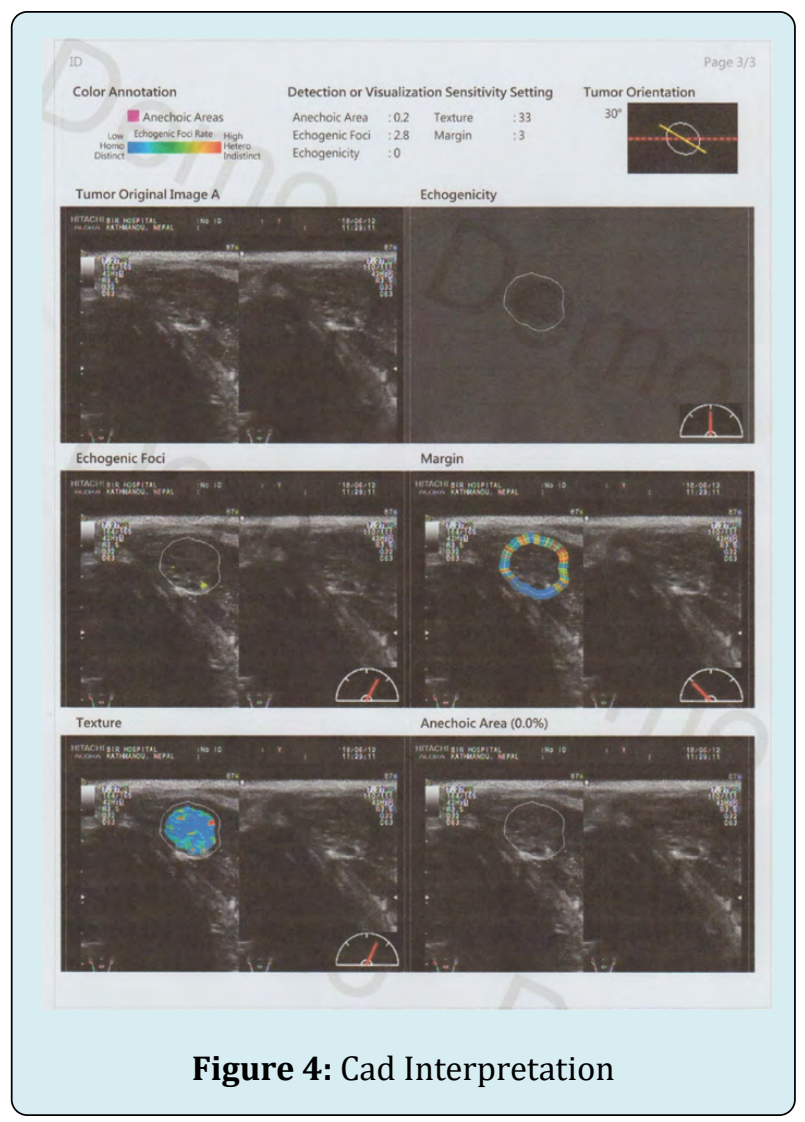

\section{Result}

Total no. of patients= 103 (Male- 24\% (25); Female- 76\%) (78)

- $\quad$ Mean Age -SD $45 \pm 12.6$

- $\quad$ Age range $=20-82$ years
- $\quad$ Common age group $=26-45$ years $(52.4 \%)$

- Duration of illness $=6$ months to 25 years More frequency 6 mo. to 1 year.

\begin{tabular}{|c|c|}
\hline Nodule feature & Percentage \\
\hline$>\quad$ No of Nodule & \\
\hline Solitary & $68 \%$ \\
\hline Multiple & $32 \%$ \\
\hline$\quad$ Size & $50 \%$ \\
\hline $5-10 \mathrm{~mm}$ & $50 \%$ \\
\hline$>10 \mathrm{~mm}$ & \\
\hline Calcification & $55 \%$ \\
\hline Present & $45 \%$ \\
\hline Absent
\end{tabular}

Table 1: Features of Thyroid nodules.

\section{Right lobe $45 \%$}

Left lobe $36 \%$

\section{Both lobe $19 \%$}

Two patients (25yrs \& 53 yrs male) had both malignant as well as benign lesion in different nodule identified by CAD technique \& confirm with FNA.

\section{FNA or FNAB (ATA 2015)}

In suspicious cases of nodule biopsy showed Benign in $80 \%$, Malignant in 5\% however in case Suspicious for malignancyFNA showed malignancy in 50-70\%,Indeterminate $20 \%$ \& Non-diagnostic 5\%.In Indeterminate \& non-diagnostic nodule needs Molecular Diagnosis which is diagnostic than CAD Small lesion \& deep seated lesion difficult to take FNA also manage by other methods e.g. Molecular determination.

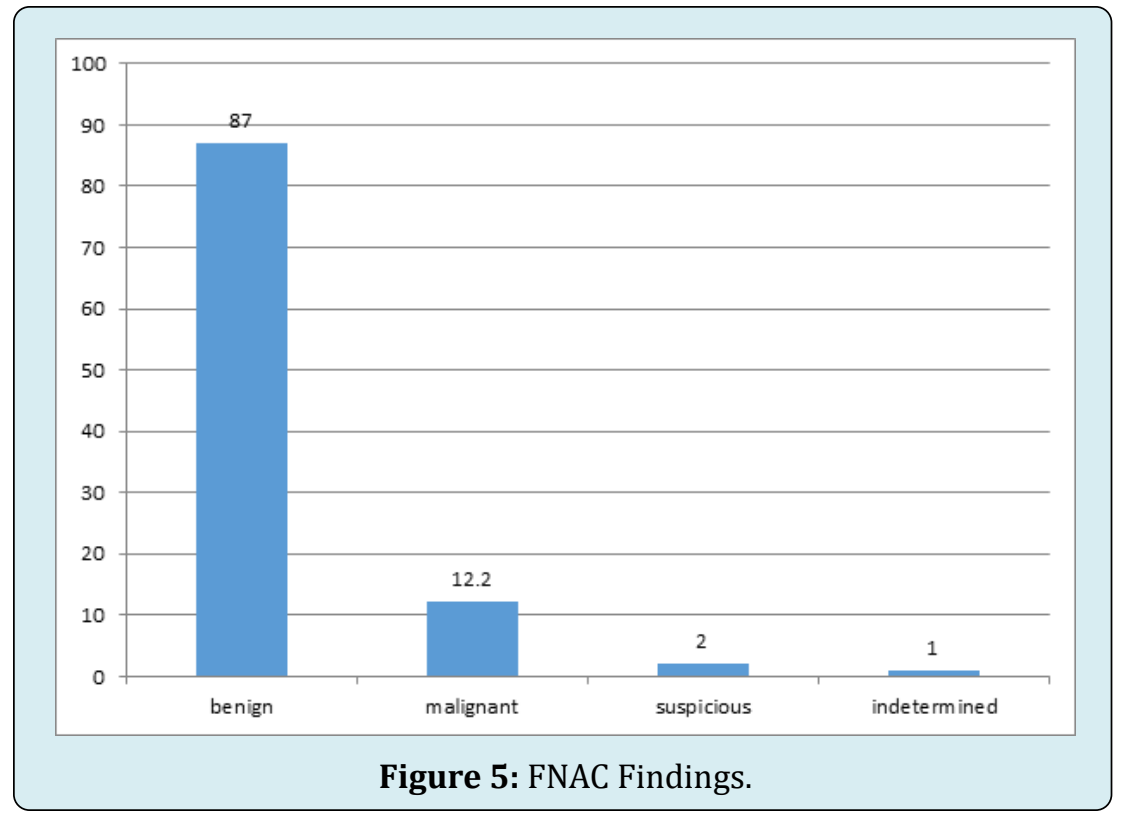




\section{Conclusions}

CAD saves work-load, has no inter-observer variation \& help to take FNA from different nodules in the same patient in suspicious cases. . However none of the examinations are perfect \& needs follow-up study. American Thyroid association (ATA) 2015 guideline suggest use of Sonographic CAD assessments of nodule to make decision for diagnostic FNA to differentiate benign from malignant nodules [6]. It has high sensitivity for detecting thyroid malignancy \& can be efficiently used for early detection of thyroid malignancy $[8,9]$. CAD is easy, accurate \& reduces the rate of FNA hence save time, money \& surgical Procedure. It is non-invasive, non-ionizing radiation procedure but it is more dependent on size \& presence of calcification hence can reduce FNA by $32 \%$ only. USG imaging interpretation may vary among the performing Radiologists but with the use of CAD, less examiner dependent. Micro-Calcification, hypo echogenicity, Ill-defined margin, Taller than wider had high risk of malignancy. Thus combination of CAD software with USG increased rate of early diagnosis. ATA 2015 guideline suggests use of Sonographic CAD assessments of nodule to make decision for diagnostic FNA to differentiate benign from malignant nodules [7].

\section{References}

1. David Sutton (1998) Text book of radiology \& imaging. Thyroid scan 46: 1287-1288.

2. Lim KJ, Choi CS, Yoon DY, Chang SK, Kim KK, et al. (2008) Computer-aided diagnosis for the differentiation of malignant from benign thyroid nodules on ultrasonography. Amcad Radiol 15(7): 853-858.

\section{Clinical Radiology \& Imaging Journal}

3. Nachiappan AC, Metwalli ZA, Hailey BS, Patel RA, Ostrowski ML, et al. (2014) The thyroid: review of imaging features and biopsy techniques with radiologicpathologic correlation. Radiographic 34(2): 276-293.

4. Chang Y, Paul AK, Kim N, Baek JH, Choi YJ, et al. (2016) Computer-aided diagnosis for classifying benign versus malignant thyroid nodules based on ultrasound images : A comparison with radiologist-based assessments. Med Phys 43(1): 554.

5. Koundal D, Gupta S, Singh S (2012) Computer-Aided Diagnosis of Thyroid Nodule: A Review. Int journal Computer Science Eng Survey 3(4): 67-83.

6. Cooper DS, Doherty GM, Haugen, Kloos RT, Lee SL, et al. (2009) Revised American Thyroid Association management guidelines for patients with thyroid nodules and differentiated thyroid cancer. Thyroid 19(11): 1167-1214.

7. Food and Drug Administration (2012) Guidance for Premarket Notification Submission for Computer Assisted Detection Devices. United States Food and Drug Administration.

8. Chang TC (2010) Ultrasonic features of thyroid cancers and benign thyroid nodules for determining the necessity of needle aspiration cytology. J Med Ultrasound 18: 5461.

9. (2016) The American Thyroid Association Guidelines Task Force on Thyroid Nodules and Differentiated Thyroid Cancer. Thyroid 2(6): 1-133. 